NOTA

\title{
ESTIMATIVA DO TEOR DE ÁGUA NO SOLO UTILIZANDO DADOS DE RADAR DE ABERTURA SINTÉTICA ${ }^{(1)}$
}

\author{
O. A. MÁXIMO(2), A. M. SAAD ${ }^{(3)}$ \& H. H. KUX(4)
}

\begin{abstract}
RESUMO
Em virtude da crescente demanda mundial por alimentos, um monitoramento eficaz e em larga escala da umidade do solo constitui fator de grande i mportância para a previ são de safras. Este trabal ho teve por objetivo apresentar uma técnica para o cálculo do teor de água no solo, utilizando modelos preditivos de umidade do solo, baseados em dados de radar de abertura sintética (SAR). Foram utilizados dados do SAR a bordo do J ERS-1 ("J apanese Earth Resources Satellite") e dois modelos empíricos. $O$ primeiro relaciona o coeficiente de retroespalhamento com a permissividade complexa (modelo de Dubois), e o segundo relaciona a permissividade complexa com o teor de água do solo e algumas de suas características físico-hídricas, tais como percentagem de areia e argila (modelo de Hallikainen). Inicialmente, os dados do SAR/J ERS-1 foram calibrados e, por meio do modelo de Dubois, foram calculados os valores de permissividade complexa. Para tanto, foi necessário inserir níveis estimados de rugosidade do solo. A partir destes resultados, utilizou-se o modelo de Hallikainen para calcular a umidade volumétrica. A análise geral dos resultados indica que a técnica de estimação de umidade do solo a partir de imagens de radar de abertura sintética, utilizada neste estudo, mostrou-se física e matematicamente exeqüível. No entanto, apresentou uma precisão moderada, não sendo ainda recomendada para o uso operacional no mapeamento de umidade do solo. A análise dos resultados revel ou também que a preci são dos dados é bastante influenciada pela precisão dos valores de rugosidade introduzidos.
\end{abstract}

Termos de indexação: umidade do solo, milho, modelos preditivos.

(1) Parte da Tese de Mestrado do primeiro autor, apresentada e aprovada no INPE, São J osé dos Campos (SP), em 21 de outubro de 1997. Recebido para publicação em julho de 1998 e aprovado em fevereiro de 1999.

(2) Pesquisador da Divisão de Sensoriamento Remoto do Instituto de Estudos Avançados do Centro Técnico Aeroespacial. Rodovia dos Tamoios, Km 5,5, CEP 12228-840 SãoJ osé dos Campos (SP). E-mail: maximo@ieav.cta.br.

(3) Pesquisador colaborador do Instituto de Pesquisas Tecnológicas - IPT, São Paulo, em pós-doutoramento no Departamento de Engenharia Rural - DER da Escola Superior de Agricultura “Luiz de Queiroz"- ESALQ-USP. Av. Pádua Dias, 11, Caixa Postal 09, CEP 13418-900 Piracicaba (SP). Email: amsaad@carpa.ciagri.usp.br.

(4) Pesquisador do Instituto Nacional de Pesquisas Espaciais, Caixa Postal 515, CEP 12.227-010 São J osé dos Campos (SP). E-mail: hermann@tid.inpe.br. 


\title{
SUMMARY: SOIL MOISTURE RETRIEVAL USING SAR DATA
}

\begin{abstract}
This paper descri bes a techniquethat retrieves soil moisturefrom monopol arized SAR data. The technique uses SAR data obtained by the J apanese Earth Resources Satelite (J ERS-1) and two empirical models. Onere ates thebackscatter coefficient with thecomplex permitivity (Dubois's model), and the other relates the complex permitivity with soil parameters such as soil moisture (Hallikainen's moded). First, theSAR data werecalibrated to obtain the backscatter coefficient and the Dubois's mode was inverted to achieve the compl ex permitivity. Theinsertion of estimated values of roughness was necessary to use the inverted equation with J ERS-1SAR data. Using theseresults and the Hallikainen's model, the soil moisture was cal culated and compared with field measurements. Theresults were found to bemoderately accurateand theinverted modes s werequitesensitiveto theprecision of the estimated values of roughness.
\end{abstract}

Index terms: synthetic apertureradar, water content, corn, retrieval models.

\section{INTRODUÇÃO}

No Brasil, as grandes extensões de áreas irrigadas (400.000 ha) estão localizadas no CentroOestee noSudeste, em regiões decerrado. Essa área está sendo irrigada por meio do sistema de aspersão mecanizado, cujo equipamento de maior expressão éo pivô central. O consumo anual de água estimado nessa área é de $1,12 \times 10^{9} \mathrm{~m}^{3}$. Na totalidade, não existe nenhum sistema de controle da quantidade deágua a ser aplicada, tampouco definido o momento ideal para se iniciar a irrigação. Esse fato faz com que a eficiência do uso do recurso hídrico de superfície seja muito inferior à deáreas semel hantes em países onde essa tecnologia é muito difundida, por exemplo, no estado de Nebraska, nos Estados Unidos da América. A busca de uma solução para minimizar o problema do controle da água de irrigação em grande escal a constitui grande desafio tecnológico na área de geoprocessamento. Assim sendo, a utilização de técnicas de sensoriamento remoto, embora ainda não completamente operacionais, tem possibilitado grandes avanços no campo da agricultura irrigada, pois fornece meios para obter informações numa escala ampla, possibilitando uma análise mais global do problema.

Empregando as técnicas de sensoriamento remoto, as faixas do espectro el etromagnético geralmente utilizadas na estimativa de umidade do solo são as do visível, infravermelho termal e microondas (I dso et al., 1975). Dentre estas, a faixa das mi croondas vem sendo cada vez mais explorada pelas suas características particulares, tais como a capacidade de penetrar nuvens e chuva e sua independência da luz solar (I dso et al., 1975). Além disso, segundo Schmugge (1983), a quantidade de água no solo influi diretamente no padrão de respostas do solo, quando imageado por sensores que operam na faixa demi croondas. Essa influência está baseada no fato de quea constante dielétrica da água é de aproximadamente 80, enquanto a do solo seco fica entre 3 e 5.

Nos últimos anos, diversos trabal hos vêm sendo desenvol vidos para modelar a influência da umi dade nas propriedades termais e dielétricas do solo, bem como para monitorar a quantidade de água no solo por meio do sensoriamento remoto por microondas. Exemplos destes trabalhos são os de Ulaby et al. (1978, 1979); Chang et al. (1980); Wang \& Schmugge (1980); Dobson \& Ulaby (1981); Dobson et al. (1985).

Dentro desse contexto, este trabalho teve por objetivo apresentar uma técnica para o cálculo do teor de água no solo, utilizando modelos preditivos de umidade do solo, baseados em dados de radar de abertura sintética.

\section{Teoria}

Segundo Ulaby et al . (1981), todo dispositivo que opera na faixa de mi croondas (radiação com freqüência entre 0,3 e $300 \mathrm{GHz}$ ), no qual um transmissor ilumina uma superfície e um receptor capta e mede al gumas propriedades do sinal que retorna aosensor proveniente dessa superfície, pode ser denominado RADAR (Radio Detection and Ranging).

Um radar imageador é um dispositivo que gera uma imagem a partir dos sinais refletidos pela superfície iluminada. Tais sinais são chamados de ecos de radar.

Nesses radares, que, em geral, têm a geometria de imageamento ilustrada na figura 1 , a imagem é produzida por meio do movimento da plataforma onde o radar está instalado sobrea área deinteresse. Um pulso curto é transmitido pelo radar, propagando-se da linha de visada mais próxima até a mais distante, numa seqüência de transmissões e recepções de pulsos (Damião, 1994). 


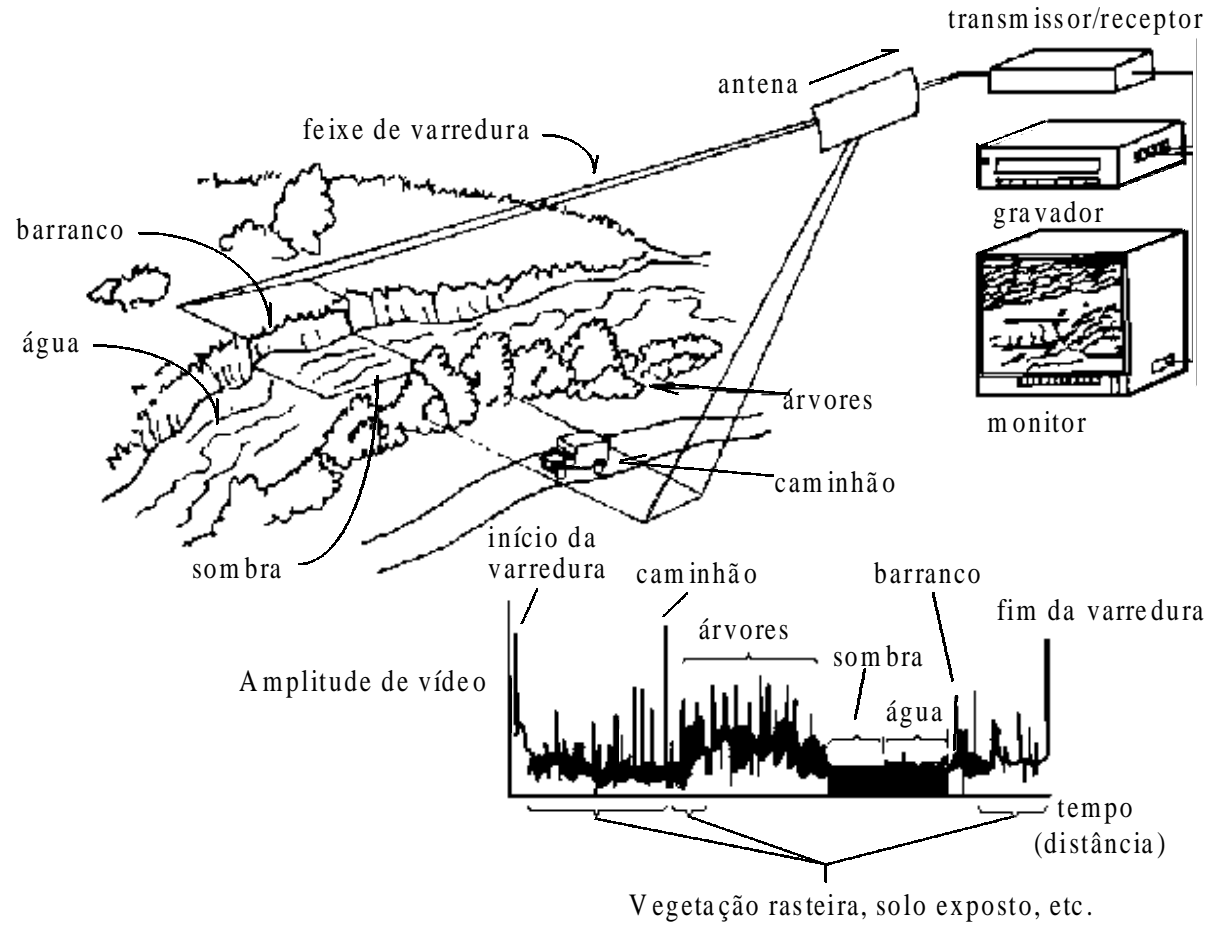

Figura 1. Geometria de aquisição de imagens de radar.

Quando o pulso incide sobre o alvo, parte do sinal retorna ao radar. O eco devido a cada pulso transmitido é amostrado, digitalizado egravado em memória, fita ou outro meio físico de armazenamento de dados. A seqüência dos pulsos é ajustada para estar em sincronismo com o movimento da plataforma. Assim, quando esta se mover o equivalentea uma largura de feixe, osinal de retorno será proveniente de uma faixa do solo diferente e adjacente. A seqüência de sinais formada com o desl ocamento da plataforma gera a imagem do radar (Ulaby et al., 1982).

Pode-se perceber que a distinção entre diferentes objetos sensoriados é causada principalmente pela diferença na potência do sinal recebido pelo radar. Desse modo, a medição precisa da potência desse sinal é de grande importância. A relação fundamental entre as características do alvo, do radar e do sinal recebido é expressa por meio da "E quação do Radar" (Ulaby et al., 1982).

Segundo Skolnik (1962), uma das formas clássicas de se apresentar esta equação é:

$$
P_{r}=\frac{P_{t} A_{e}^{2}}{4 \pi \lambda^{2} R^{4}} \cdot \sigma
$$

em que

$P_{r}$ éa potência que retorna ao radar em $W$, $P_{t}$ é a potência de transmissão em $W$,

$A_{e}$ é a área efetiva de recepção da antena em m², $\lambda$ éo comprimento de onda da radiação usada em $\mathrm{m}$, $R$ é a distância sensor-superfície em $m$ e $\sigma$ éo coeficiente de retroespal hamento do al vo em $\mathrm{m}^{2}$.

Uma vez escol hido o sensor, todos os parâmetros desta equação, com exceção do coeficiente de retroespal hamento, tendem a permanecer constantes, de modo que as variações na potência de retorno devem-se, primordialmente, a variações nos val ores do coeficiente de retroespal hamento.

Assim, se o coeficiente de retroespal hamento de um alvo puder ser modelado em função de al gumas de suas características (rugosidade, constituição química eumi dade, por exemplo), pode-seestabel ecer uma relação entre os dados do radar e estas características do alvo.

Para modelar o coeficiente de retroespal hamento a partir de parâmetros da área iluminada, duas abordagens têm sido utilizadas: uma abordagem teórica e outra empírica. A abordagem teórica é composta por equações que, muito embora sejam úteis para explicar diversos fenômenos, têm, na maioria das vezes, uma região de validade muito restrita, induem variáveis difíceis deser encontradas e de difíal implementação computacional. Ulaby et al. $(1982,1986)$ apresentaram uma descrição das formulações de Kirchoff, que descrevem teoricamente - espalhamento de superfícies segundo uma aproximação escalar (Modelo deÓptica Física) euma aproximação de fase estacionária (Model o deÓptica Geométrica). 
Para contornar as restrições impostas pelos model os teóricos, utiliza-se a abordagem empírica, que modela o coeficiente de retroespalhamento em função de poucos e simples parâmetros, tais como a rugosidade, o teor de água e o ângulo de incidência. Diversos trabalhos vêm sendo realizados com esse objetivo, dos quais se destacam os de Oh et al. (1992, 1994), Ulaby \& Siquiera (1995) eDubois et al. (1995).

\section{MATERIAL E MÉTODOS}

A área de estudo escolhida foi um pivô central, localizado na Fazenda Cachoeirinha, município de Guaíra (20²7'30"S, 48¹9'30’W, 495 m) no estado de SãoPaulo.

Este pivô apresenta 570 m de raio e, na época do estudo (setembro de 1995), estava subdividido em quatro quadrantes, nos quais havia plantações de milho, com 5, 20 e 135 dias de germinação, e soja, com 30 dias de germinação. F oram escol hidas as áreas de milho com cinco dias após a emergência, para representar solo exposto, ea área de mil ho com 20 dias, para avaliar a influência da cobertura vegetal na precisão dos resultados. Os quadrantes restantes foram desconsiderados por impedirem a medição da rugosidade, considerando a estrutura do dossel.
A unidade pedológica encontrada nessa região é o Latossol o Roxo. O quadro 1 apresenta os valores médios para um perfil de 0-0,5 $\mathrm{m}$ de alguns parâmetros físicos desse solo da fazenda Cachoeirinha, fornecidos pelo I nstituto de Pesquisas Tecnológicas.

A coleta de dados foi efetuada de forma radial, como mostra a figura 2. Em cada ponto, foram coletadas amostras de rugosidade e de umidade. Foram realizadas, ao todo, 34 medidas na área de interesse. Desse total, 24 leituras foram feitas na área de milho com cinco dias de emergência e as restantes (10 leituras) na área de mil ho com 20 dias de emergência.

Para determinar a rugosidade, foi utilizado um rugosímetro de varetas, desenvolvido na Universidade deWageningen (Holanda) e construído nas oficinas do Instituto Nacional de Pesquisas Espaciais (INPE). Para determinar a umidade, as amostras foram col hidas na camada de 0-0,05 m e foi utilizado o método de estufa (105 a 115C).

O trabalho de campo foi realizado no mesmo horário em quea região estava sendo imageada pelo "J apanese Earth Resources Satellite" (J ERS-1). O quadro 2 apresenta as principais características desse satélite.

Segundo Nemoto et al. (1991), oradar de abertura sintética a bordo doJ ERS-1 opera na freqüência de

Quadro 1. Características físicas do Latossolo Roxo A moderado textura muito argilosa, Fazenda Cachoei rinha, Guaíra, SP. Valores médios para perfil de 0-0,5 m

\begin{tabular}{|c|c|c|c|c|c|c|c|c|c|c|}
\hline \multicolumn{7}{|c|}{ Granulometria (g kg-1) } & \multirow{2}{*}{\multicolumn{2}{|c|}{ Densidade (kg dm-3) }} & \multirow{2}{*}{\multicolumn{2}{|c|}{ Porosidade $\left(\mathrm{m}^{3} \mathrm{~m}^{-3}\right)$}} \\
\hline \multicolumn{5}{|c|}{ Areia } & \multirow{2}{*}{ Silte } & \multirow{2}{*}{ Argila } & & & & \\
\hline MG & G & $\mathbf{M}$ & $\mathbf{F}$ & MF & & & Solo & Partícula & Calculada & Determinada \\
\hline 0,3 & 1,5 & 7,8 & 10,3 & 5,0 & 18,8 & 56,5 & 1,22 & 3,04 & 62,5 & 57,5 \\
\hline
\end{tabular}

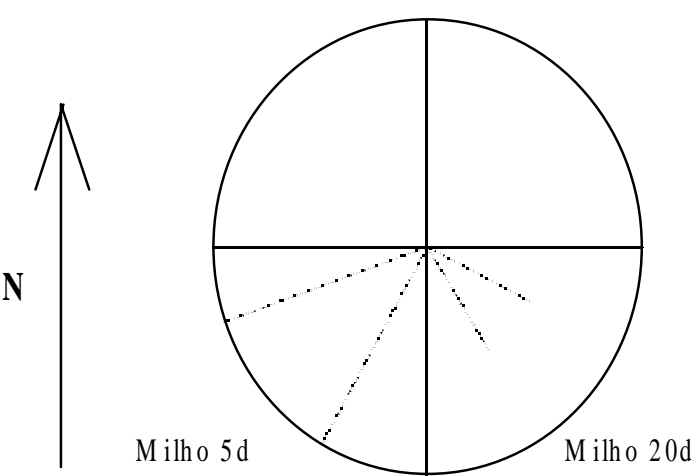

Figura 2. Desenho esquemático da coleta de dados.
Quadro 2. Características técnicas do J ERS-1

\begin{tabular}{ll}
\hline Órbita & Sol-síncrona \\
Altitude & $568 \mathrm{Km}$ \\
Período & $96 \mathrm{~min}$ \\
Período de recorrência & 44 dias \\
Horário de passagem asc. & $10: 30$ até 11:00 horário local \\
& - Radar de Abertura Sintética \\
Equipamentos da missão & - Sensor óptico \\
& - Gravador de dados \\
Veículo lançador & - Transmissor de dados \\
\hline
\end{tabular}

Fonte: NASDA (1994). 
1.275 M Hz, polarização horizontal para envio e recepção e gera imagens com uma resolução espacial de 18 metros.

A figura 3 apresenta a imagem utilizada no trabal ho com o pivô em destaque.

O organograma esquemático para calcular a umidade do solo a partir de uma imagem de radar encontra-se na figura 4.

Para esse cál culo, dividiu-se o trabal ho em duas etapas. Na primeira, foi feito o trabalho de campo ao mesmo tempo em que a área era imageada pelo radar a bordo doJ ERS-1. A partir da imagem obtida pelo sensor, foi realizada a extração do valor digital dos pixels da área selecionada e, posteriormente, a conversão desses val ores para val ores de coeficiente de retroespalhamento $(\sigma$ ) $)$.

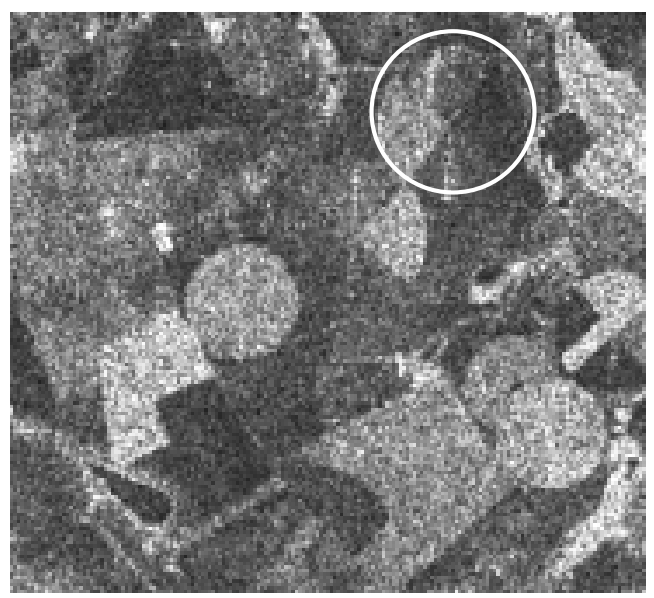

Figura 3. Área-teste com pivô em destaque.

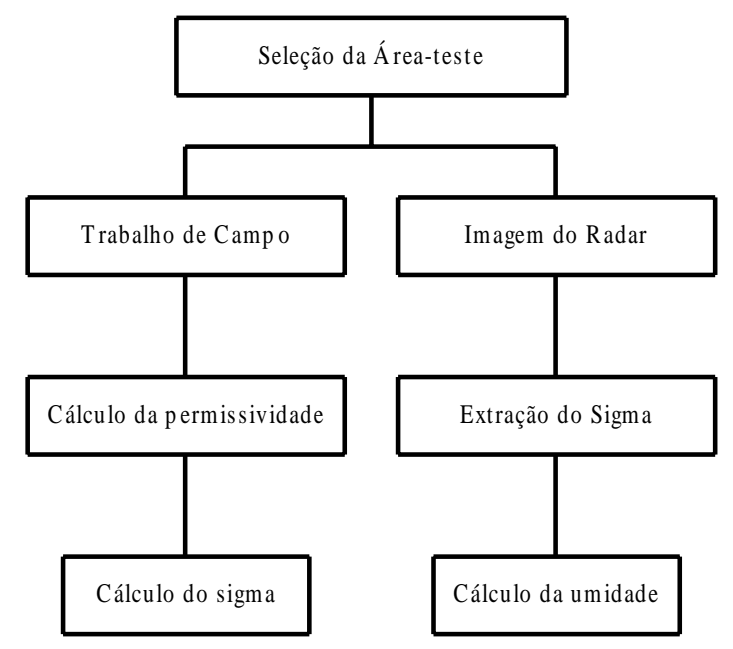

Figura 4. Organograma esquemático do desenvol vimento do cálculo da umidade do solo a partir de uma imagem de radar.
Para essa conversão, foi utilizada a relação proposta por Shimada (1996):

$$
\sigma^{\mathrm{O}}(\mathrm{dB})=\mathrm{CF}(\mathrm{dB})+10 \log \left(\frac{\sum \mathrm{VA}^{2}}{\mathrm{~N}}\right)
$$

em que

$\sigma^{\circ}$ é o coeficiente de retroespal hamento em dB,

CF é um fator de calibração em dB,

VA é o valor do pixel e

$\mathrm{N}$ é o número de pixels.

Em seguida, os dados col etados em campo foram utilizados como parâmetros de entrada do modelo de Dubois para polarização horizontal (Equação 3), e os resultados desse cálcul o foram comparados com os obtidos da imagem.

As equações do model o de Dubois, apresentadas em Dubois et al. (1995), são:

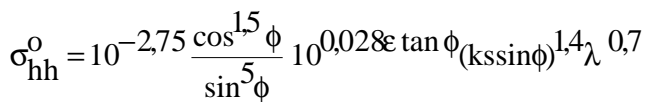

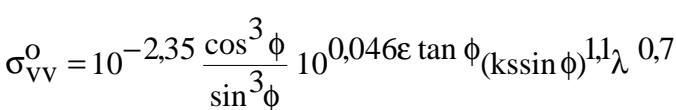

em que

$\phi$ é o ângulo de incidência em graus,

k é o número de onda,

s é o desvio-padrão de altura (altura rms),

$\varepsilon$ é a permissividade complexa e

$\lambda$ é o comprimento de onda em $\mathrm{m}$.

Na segunda etapa, os dados calculados a partir da imagem foram utilizados como parâmetros de entrada para a equação 3 e esta foi resolvida para se obterem os val ores de permissividade complexa. Em seguida, esses val ores de permissividade foram usados como parâmetros de entrada do modelo de Hallikainen, calculando-se a umidade do solo.

O model o utilizado nesse estudo, apresentado em Hallikainen et al. (1985), correspondea:

$$
\varepsilon=\left(a_{0}+a_{1} S+a_{2} C\right)+\left(b_{0}+b_{1} S+b_{2} C\right) \theta++\left(c_{0}+c_{1} S+c_{2} C\right) \theta^{2}
$$

em que

$\varepsilon$ é a permissividade complexa,

a, b e c são coeficientes empíricos conhecidos,

$S$ é a percentagem de areia do solo,

C é a percentagem de argila do solo e

$\theta$ é umidade volumétrica do solo em \%.

\section{RESULTADOS E DISCUSSÃO}

O quadro 3 apresenta um resumo dos dados de umidade volumétrica erugosidade col etados durante o trabalho de campo. 
Quadro 3. Resumo estatístico dos dados coletados em campo, na profundidade de 0-0,05 m

\begin{tabular}{|c|c|c|c|c|c|c|c|c|c|c|c|c|c|c|c|}
\hline \multicolumn{8}{|c|}{ Milho- 5 dias } & \multicolumn{8}{|c|}{ Milho - 20 dias } \\
\hline \multicolumn{4}{|c|}{ Umidade volumétrica $\left(\mathrm{m} \mathrm{m}^{-3}\right)$} & \multicolumn{4}{|c|}{ Rugosidade (rms) $)^{(1)}$} & \multicolumn{4}{|c|}{ U midade volumétrica $\left(\mathbf{m} \mathbf{~ m}^{-3}\right)$} & \multicolumn{4}{|c|}{ Rugosidade (rms) } \\
\hline Mínima & Máxima & Média & D P & Mínima & Máxima & Média & DP & Mínima & Máxima & Média & D P & Mínima & Máxima & Média & D P \\
\hline 26,6 & 32,1 & 28,6 & 1,1 & 0,82 & 2,67 & 1,36 & 0,47 & 29,4 & 30,5 & 30,0 & 0,37 & 1,27 & 2,14 & 1,61 & 0,26 \\
\hline
\end{tabular}

(1) rms representa o desvio-padrão da variação da rugosidade da superfície.

Os baixos valores de desvio-padrão (DP) indicam uma tendência de uniformidade tanto na área de milho com cinco dias de germinação quanto na área de milho com 20 dias. Essa uniformidade já era esperada por causa da agricultura irrigada desenvolvida na região (pivô central).

Esses dados, juntamente com os apresentados no quadro 1, foram utilizados no cálculo da umidade volumétrica, conforme descrito na seção anterior.

A comparação entre os valores de umidade calculados e coletados em campo é apresentada na figura 5.

Quanto à presença da vegetação, nota-se uma tendência de subestimação dos valores de umidade para a área de milho com 20 dias. Essa tendência também foi observada em Dubois et al. (1995).

A região em destaque no gráfico da figura 5 corresponde aos valores que mostram um erro máximo de $4 \%$ em relação aos val ores medidos. Esse erro é compatível com os erros médios obtidos por Oh et al. (1992) - 4\% - e Dubois et al. (1995) - 4,5\%.

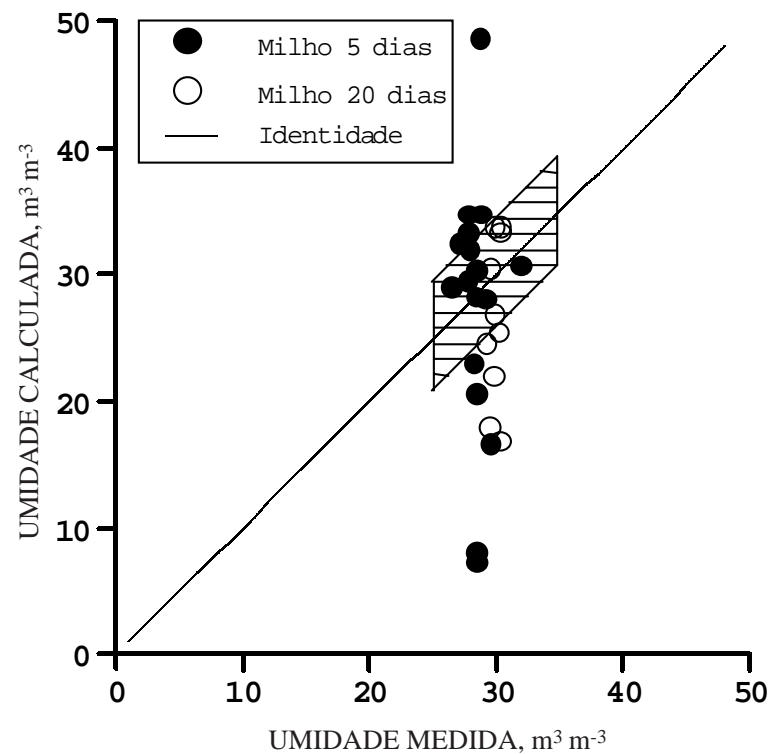

Figura 5. Comparação entre os valores de umidade calculados e medidos em campo.
Neste estudo, o erro médio para a região de milho com cinco dias foi de 7,26\% e o desvio-padrão de 7,09. Para a região de mil ho com 20 dias, o erro médio foi de 5,73\% e o desvio-padrão de 4,17.

Esses valores foram influenciados principalmente por dois fatores. O primeiro deve-se ao uso deapenas uma imagem, enquanto os modelos de Oh et al. (1992), Dubois et al. (1995) eUlaby \& Siqueira (1995), por exemplo, utilizaram duas ou mais imagens com polarizações cruzadas (HH, VV, HV, VH).

Soares \& Rennó (1996) verificaram uma queda de correlação nos resultados, quando, utilizando apenas uma imagem, obtiveram valores compatíveis com os deste estudo.

Além disso, a inserção dos val ores de rugosidade também serviu como fonte de erros no processo de determinação da umidade por meio de imagens de radar de abertura sintética. Tal influência também foi observada em Soares \& Rennó (1996).

Para que se pudesse obter uma estimativa dessa influência, foram cal culados os valores de rugosi dade que garantiriam um acerto de $100 \%$ na estimativa da umi dade, e, em seguida, introduzi dos erros nesses valores, comparando os resultados com eles e com os originais. Um resumo dos cálculos para área de milho com cinco dias é apresentado na figura 6 , onde se observa grande perda de correl ação com a inserção de erros.

A análisegeral dos resultados indica que a técnica de estimação de umi dade do sol o a partir de imagens de radar de abertura sintética, utilizada neste estudo, mostrou-se física e matematicamente exeqüível. No entanto, apresentou uma precisão moderada, não sendo ainda recomendada para o uso operacional no mapeamento de umidade do solo.

Além disso, a coleta de dados foi realizada na camada de 0-0,05 m, o que limita as conclusões para esta faixa de profundidade. É necessário que sejam realizados novos experimentos em outras profundidades para estender as conclusões para culturas que tenham uma profundidade de raízes maior.

Por fim, caberessaltar quea técnica apresentada neste estudo poderá ser aprimorada a partir de um delineamento experimental que cubra uma faixa de 


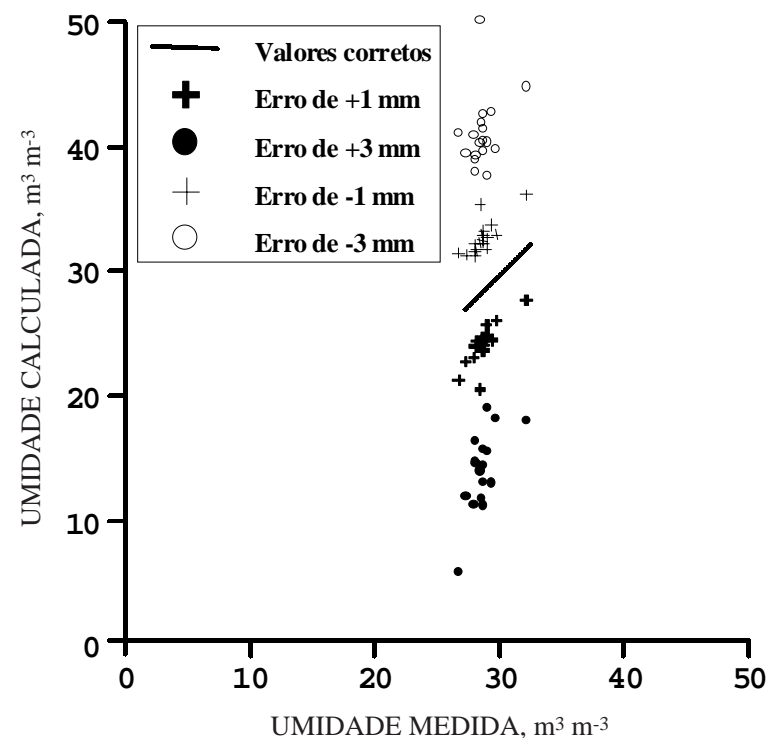

Figura 6. Influência dos erros de altura rms na correlação dos valores calculados e medi dos de umidade volumétrica.

umidade mais ampla (Quadro 3), da utilização de pares de imagens com polarizações cruzadas e do desenvolvimento de novas técnicas para a estimativa/medição da rugosidade superficial.

\section{CONCLUSÕES}

1. Segundo resultados deste trabalho, a estimativa da umidade do solo utilizando imagens de radar mostrou-se física e matematicamente exeqüível.

2. A utilização de imagens monopolarizadas exigiu a inserção de valores estimados ou medidos de rugosidade.

3. Nesse caso, a precisão da estimativa de umidade foi considerada moderada.

4. A precisão dos dados de rugosidade introduzidos revel ou forte influência na correlação dos resultados.

5. Esta técnica ainda não pode ser recomendada para um mapeamento operacional da umidade do solo ou como método de controle de manejo de irrigação.

\section{AGRADECIMENTOS}

Os autores agradecem o apoio do Instituto de Pesquisas Tecnológicas, do Instituto Nacional de Pesquisas Espaciais edoCentroTécnico Aeroespacial.

\section{LITE RATURA CITADA}

CHANG, A.T.C.; ATWATER, S.G.; SALOMONSON, V.V.; ESTES, J .S.; SIMONETT, D.S. \& BRYAN, M.L. L-Band radar sensing of soil moisture. IEEE Trans. Geosci. Remote Sens., GE18:303-310, 1980.

DAMIÃO, D.P. Análise comparativa de imagens multisensores (TM/Landsat eSAR/ERS-1) e multitemporais, na região de Guaíra, SP. São J osé dos Campos, Instituto Nacional de Pesquisas Espaciais, 1994. 107p. (Tese de Mestrado)

DOBSON, M.C. \& ULABY, F.T. Microwave backscatter dependence on surface roughness, soil moisture, and texture: Part III - soil tension. IEEE Trans. Geosci. Remote Sens., GE-19:51-61, 1981.

DOBSON, M.C.; ULABY, F.T.; HALLIKAINEN, M.T. \& ELRAYES, M.A. Microwave dieletric behavior of wet soil - Part II: dieletric mixing models. IEEE Trans. Geosci. Remote Sens., GE-23:35-46, 1985.

DUBOIS, P.C.; ZYL, J . \& ENGMAN, T. Measuring soil moisture with imaging radars. IEEE Trans. Geosci. Remote Sens., 33:915-926, 1995.

HALLIKAINEN, M.T.; ULABY, F.T.; DOBSON, M.C. \& ELRAYES, M.A. Microwave diel etric behavior of wet soil - Part I: Empirical models and experimental observations. IEEE Trans. Geosci. Remote Sens., GE-23:25-34, 1985.

IDSO, S.B.; J ACKSON, R.D. \& REGINATO, R.J . Detection of soil moisture by remotesurveillance. Am. Sci., 63:549-557, 1975.

NASDA - Earth Observation Center. JERS-1 Data users handbook. 296p. 1994.

NEMOTO, Y.; NISHINO, H.; ONO, M.; MIZUTAMARI, H.; NISHIKAWA, K. \& TANAKA, K. J apanese earth resources satellite- 1 - Synthetic Aperture Radar. Proc. IEEE, 79:800809, 1991.

OH, Y.; SARABANDI, K. \& ULABY, F.T. An empirical model and an inversion technique for radar scattering from soil surfaces. IEEE Trans. Geosci. Remote Sens., GE-30:370381, 1992.

OH, Y.; SARABANDI , K . \& ULABY, F.T. An inversion al gorithm for retieving soil moisture and surface roughness from polarimetric radar observation. In: INTERNATIONAL GEOSCI. REMOTE SENS. SYMPOSIUM, 1994, Pasodena, CA, Proceedings. IEEE Geoscience and Remote Sensing Society (GRSS), 1994. CD-ROM

SCHMUGGE, T.J. Remote sensing of soil mosture: recent advances. IEEE Trans. Geosci. Remote Sens., GE-21:336344, 1983.

SHIMADA, M. Radiometric and geometric calibration of J ERS1 SAR. Adv. Space Res., 17:79-88, 1996.

SKOLNIK, M.L. Introduction to radar systems. 2.ed. New York, McGraw-Hill, 1962. 648p.

SOARES, J.V.\& RENNÓ, C.D. Soil moisture retrieval from active microwave remote sensing. In: LATINO-AMERICAN SEMINAR ON RADAR REMOTE SENSING, 1., 1996, Buenos Aires, Proceedings. Buenos Aires, 1996. p.195-203.

ULABY, F.T.; BATLIVALA, P.P. \& DOBSON, M.C. Microwave backscatter dependence on surface roughness, soil moisture, and texture: Part I - bare soil. IEEE Trans. Geosci. Elec., GE-16:286-295, 1978 
ULABY, F.T.; BRADLEY, G.A. \& DOBSON, M.C. Microwave backscatter dependence on surface roughness, soil moisture, and texture: Part II - vegetation covered soil. IEEE Trans. Geosci. Elec., GE-17:33-40, 1979.

ULABY, F.T.; MOORE, R.K. \& FUNG, A.K. Microwave remote sensing - Active and passive. Washington, D.C., Artech House, v.1. 456p. 1981.

ULABY, F.T.; MOORE, R.K. \& FUNG, A.K. Microwave remote sensing- Active and passive. Washington, D.C., Artech House, v.2. 1064p. 1982.
ULABY, F.T.; MOORE, R.K. \& FUNG, A.K. Microwave remote sensing- Active and passive. Washington, D.C., Artech House, v.3. 1097p. 1986.

ULABY, F.T. \& SIQUEIRA P. Polarimetric SAR soil moisture inversion algorithms. East Lansing, University of Michigan, 1995. (Technical Memorandum)

WANG, J .R. \& SCHMUGGE, T.J . An empirical model for the complex dieletric ermitivity of soils as a function of water content. IEEE Trans. Geosci. RemoteSens., GE-18:288-295, 1980. 